\title{
EL NUEVO MUNDO: UNA RUTA PARA SU COMPRENSIÓN EN LA HISTORIA GENERAL DE FRAY BERNARDINO DE SAHAGÚN
}

Pilar Máynez

Universidad Nacional Autónoma de México (UNAM), México

pilar_unam@hotmail.com

\section{DOI: https://doi.org/10.26512/caleidoscopio.v2i1}

RESUMEN: El encuentro del Nuevo Mundo trajo como consecuencia la traducción de su entorno y de los elementos que conformaban la cultura de sus habitantes a partir de la perspectiva particular del intérprete. En este trabajo se aborda concretamente la manera en que el franciscano Bernardino de Sahagún (14991590), a través de diferentes estrategias lingüísticas y desde su visión europea y renacentista, definió las muy diversas realidades que integraban la cultura mexica en su Historia General de las cosas de Nueva España.

Palabras clave: Viejo Mundo, Nuevo Mundo, constructivismo, comprensión, traducción.

\section{THE NEW WORLD: A ROUTE FOR ITS UNDERSTANDING IN FRAY BERNARDINO DE SAHAGÚN'S GENERAL HISTORY}

\begin{abstract}
The encounter with the New World brought about the translation of what surrounded it and the elements that the culture of its inhabitants comprised, all according to the particular perspective of each interpreter. This paper specifically tackles the way in which the Franciscan Bernardino de Sahagún (14991590) defined the very diverse realities integrating the Mexica culture back then, that is, through different linguistic strategies and from its Renaissance, European vision, both reflected in his General History of the Things of New Spain (Historia General de las cosas de Nueva España).
\end{abstract}

Keywords: Old World, New World, Constructivism, understanding, translation. 


\section{Introducción}

La aparición de insospechadas tierras ante los ojos de los conquistadores europeos trajo consigo la necesidad de ser designadas. ¿Cómo hacer referencia a aquellos ignotos parajes? ¿Se trataba de un mundo nuevo o, en realidad, era otro? Fray Juan de Torquemada sostenía al respecto que sólo existía un mundo, pues Dios lo había creado para el hombre y no había necesidad de más, y "con solo éste se acudía a la satisfacción del negocio" (FROST, 2002, p.139); por tanto, la denominación del Nuevo Mundo era correcta y más aún si, tomando en cuenta la disquisición aristotélica, se considera que tal entidad correspondía a todo aquello que cubre el cielo ${ }^{1}$.

Una vez aceptada la existencia de un único mundo era necesario explicar la relación de los pobladores indígenas que lo habitaban con el linaje de Adán. Fray Bernardino de Sahagún afirmaba al respecto que "es certísimo que estas gentes son nuestros hermanos, procedentes (sic) del tronco de Adam como nosotros. Son nuestros próximos, a quien somos obligados a amar como a nosotros mismos. Quid quid sit"2.

Por otra parte, algunos testimonios indígenas que aludían a un largo y penoso camino iniciático se relacionaron, como afirma Elsa Cecilia Frost, con los relatos de la historia primigenia de la humanidad que aparecía en los libros sagrados cristianos. La explicación del quehacer misionero se homologaba así con el realizado por los apóstoles, como lo narra el dominico fray Diego Durán (1538c.1588):

[...]Porque aunque me quiera atar al sagrado evangelio que dice por San Marcos que mandó Dios a sus sagrados apóstoles que fuesen por el mundo y predicasen el evangelio a toda creatura, prometiendo a los que creyesen y fuesen bautizados la vida eterna, no me osaré afirmar en que este varón fuese algún apóstol bendito. Pero gran fuerza me hace su vida

\footnotetext{
${ }^{1}$ Elsa Cecilia Frost abunda al respecto: "Por su parte, fray Juan de Torquemada refuta a quienes pudieron pensar que había muchos mundos y llega a afirmar que tal opinión no pasa de ser disparate de antiguos". Reconoce, como verdad irrefutable, que el poder infinito de Dios, así como creó un mundo, pudo crear otro y muchos, y "cuantos más fuere su santísima voluntad". Pero, se pregunta el fraile, ¿qué causa necesaria habría para que fueran muchos? Y tras de responder que ninguna, se plantea una nueva pregunta, "si no es más que un Dios ¿de qué servían muchos mundos?" (FROST, 2002,, p.139).

2 (Sahagún, 2002, T,I., p. 64).
} 
y obras a pensar que, pues estas eran creaturas de Dios racionales y capaces de la bienaventuranza que no las dejaría sin predicador, y si lo hubo, fue Topiltzin (DURÁN, 1967, p. 9-10).

Los misioneros encargados de alumbrar a los idólatras americanos, cuya tarea podría asemejarse con la emprendida por los apóstoles o con la de los doce compañeros que habían acompañado a San Francisco en su prédica, concibieron la empresa que los había traído al Nuevo Mundo como una batalla que tendrían que librar contra la serpiente antigua, pues el demonio estaba en todos aquellos cultos diferentes del cristiano.

La aparición de un Nuevo Mundo conllevó de este modo no sólo su subsecuente denominación la cual implicaba, como hemos visto, el concepto que de él se tenía y la explicación de su posible vínculo con el viejo continente. Su existencia planteó un complejo proceso designativo que representaba lo propio de aquellas tierras mediante determinadas formas lingüísticas, en fin, de las "cosas" que en él existían y que se encontraban, dispuestas en un orden específico. Como advierte Michel Foucault (1926-1984) “...nada hay más vacilante, nada más empírico (cuando menos en apariencia) que la instauración de un orden de las cosas [...] El orden es, a la vez, lo que se da en las cosas como su ley interior" (FOUCAULT, 1998, p. 5). En toda cultura, entre el uso de lo que pudiéramos llamar los códigos ordenadores y las reflexiones sobre el orden, existe una experiencia desnuda respecto a las disposiciones y sus modos de ser². En ese instante es donde aparece el poder de las palabras cuya función consiste en identificar seres y objetos, y en representar el pensamiento. De este modo, las cosas pueden ser asibles. Mediante el nombre, el mundo se torna disponible y se le puede reconocer.

\footnotetext{
3 Y continúa: "El orden es, a la vez, lo que se da en las cosas como su ley interior, la red secreta según la cual se miran en cierta forma unas a otras, y lo que no existe a no ser a través de la reja de una mirada, de una atención, de un lenguaje; y sólo en las casillas blancas de este tablero se manifiesta en profundidad como ya estando ahí, esperando en silencio el momento de ser enunciado" (FOUCAULT, 1998, p. 5).
} 


\title{
El mundo de los objetos, el mundo de las palabras y su proceso de construcción
}

El acto designativo aparece, entonces, según se mencionó anteriormente, como un modo de apropiación de las nuevas realidades indoamericanas. Ángel Rosenblat (1902-1984) lo explica de la siguiente manera:

\begin{abstract}
Los nombres de las cosas y de los lugares y la visión misional del conquistador de América representan una proyección de la mentalidad europea. Los descubridores y pobladores hicieron entrar la realidad americana en los moldes de las palabras, los nombres y las creencias de Europa. Es decir, la acomodaron a su propia arquitectura mental. Sobre el mundo americano proyectaron no sólo la realidad tangible de su mundo europeo, sino también su tradición literaria, mitología y la religión (ROSENBLAT, 1977, p. 160).
\end{abstract}

No obstante, ese patrón conceptual del que partían los europeos para asir aquel nuevo mundo que se manifestaba en sus más diversas formas y expresiones ante ellos era muy diferente, ya que como sostuvo John Beattie (1915-1990) "pues si en un sentido todos los hombres de cualquier parte habitan el mismo mundo, en otro importante sentido habitan mundos diferentes" (1980, p. 105). Lo anterior nos conduce, por tanto, a las siguientes interrogantes: ¿Puede una lengua trasladar completamente los contenidos de su cultura a otra? ¿Puede realmente un sistema lingüístico recubrir las zonas de otros espectros semánticos? Coincidimos con la premisa relativista de que el pensamiento y el lenguaje están estrechamente relacionados; también estamos de acuerdo en que es a través de las unidades lingüísticas como podemos aprehender el pensamiento - ese todo nebuloso al que sólo se accede mediante las formas lingüísticas - y que las distintas lenguas son manifestaciones de las diferentes maneras de segmentar el universo. Sin embargo, todavía no resulta muy claro el modo en que estos elementos se corresponden e interactúan, aún no se ha logrado comprobar en la totalidad de un sistema lingüístico los mecanismos de interacción con la cultura. Emile Benveniste advierte que este problema está lejos de ser sencillo y es, en efecto, el problema medular de la situación de la lengua respecto de la sociedad 4 .

\footnotetext{
${ }^{4}$ En el capítulo "Estructuras de la lengua y la sociedad" en Problemas de lingüística general II explica respecto a esta relación que: "La lengua rodea por todas partes la sociedad y la contiene en su 
Ahora bien, la manera en que construimos y organizamos los nuevos conceptos sobre las cosas que están en estrecha relación con determinados esquemas previos, nunca puede ser estática, depende de situaciones que surgen de un caso específico de lengua y de su conceptualización expuesta en eventos discursivos concretos. Así, sobre la base de nuestra propia experiencia, se modifica nuestra identidad que se transforma constantemente en virtud del entorno y de nuestras interacciones. El hombre, pues, al encontrarse en el mundo y en su circunstancia debe reconocer y clasificar las cosas que en él existen: los vegetales, los animales y otros seres humanos con los que interactúa. Lo anterior depende, entonces, del lugar que ocupa ese hombre en un contexto específico en el que existen cosas ya sea en su modo presente o latente, oculto. En ese entorno el hombre aparece como un personaje espacial, dice José Ortega y Gasset (1972, p. 104). Se puede cambiar de sitio pero cualquier que éste sea, estará vinculado íntimamente con el aquí. El yo y el aquí son inseparables y ese desde aquí se torna inevitablemente en una particular perspectiva; se trata de la ley estructural enarbolada por el filósofo español que supone el hecho de que el hombre no es sino cada cual que tiene un mundo y un aquí.

Nuestros «aquís» se excluyen, no son interpenetrables, son distintos, y
por eso la perspectiva en que le aparece el mundo es siempre distinta de
la mía. Por eso no coinciden suficientemente nuestros mundos. Yo estoy,
por de pronto, en el mío y él en el suyo. Nueva causa de soledad radical.
No solo yo estoy fuera del suyo, sino que también mi mundo está fuera
del suyo: somos, mutuamente, dos «fueras» y por eso somos
radicalmente forasteros (ORTEGA Y GASSET, 1972, p. 109).

Retomando lo mencionado anteriormente respecto a las conceptualizaciones que los hombres del Viejo Mundo fueron formándose sobre los del Nuevo de acuerdo con su particular perspectiva, existe, asimismo, una copiosa historiografía - cuyos testimonios datan del siglo XVI - que avala el complejo proceso que los nativos indomexicanos, por su parte, llegaron a tener de aquellos extraños seres que irrumpieron en su territorio. La más difundida fue aquélla que los hizo concebir como teules, según lo consignan Bernal Díaz del Castillo y Francisco de Aguilar, la cual ha estado presente en las reflexiones de instaurando lo que podría denominarse semantismo social” (BENVENISTE, 1982, p. 101-102). 
cronistas e historiógrafos. Así, por ejemplo, para Jorge Gurría Lacroix "los mexicas pensaron que, en efecto [los castellanos] eran dioses (teules), pues tenían el control del fuego y del humo" (PASTRANA, 2004, p. 69). Para este y otros autores los adelantos tecnológicos de los europeos hicieron que los indígenas les atribuyesen poderes sobrenaturales. Los aztecas, en el momento del contacto "ignoran la alteridad humana radical y, al encontrarla, utilizan la única categoría disponible, la que admite, justamente la extrañeza radica: la de los dioses" (idem). Por otra parte, también los conquistadores construyeron estructuras particulares propias de esa ignota realidad; produjeron significaciones y atribuciones de sentido las cuales fueron expresadas mediante el lenguaje, que constituyó su entrada a ese otro mundo. A través del lenguaje se pautó un estilo y se pudieron aprehender los elementos propios de ese nuevo escenario.

Ahora bien, la elaboración de modelos individuales, familiares y socioculturales que revisten la observación de cualquier individuo y propician la selección o el corte de la realidad los condujo a ver eso y no otra cosa. De ahí que podamos considerar las premisas de Ortega y Gasset, a las que hemos aludido anteriormente, como claro antecedente de la corriente constructivista, la cual postula que el mundo se crea por el que está observándolo. Como sostiene Ernest von Glasersfeld, en su introducción al constructivismo radical, el conocimiento será concebido como algo que "el organismo construye en el intento de ordenar [el] amorfo flujo de la experiencia, estableciendo experiencias repetibles y relaciones confiables entre ellas. Las posibilidades de construir ese orden están determinadas y perpetuamente constreñidas por los pasos precedentes en la construcción" (las cursivas son nuestras. Glaserfeld, https//jeasacademia.wordpress.com, recuperado 9 dic-2017).

El constructivismo plantea que una persona considerada en los aspectos cognitivos, sociales y afectivos del comportamiento no es tan sólo el resultado del ambiente ni de sus propias disposiciones internas sino una construcción particular que se va produciendo sucesivamente. Esta forma específica de concepción se genera por la representación inicial que se tiene de la nueva información y por la actividad interna o externa que desarrolla cada individuo. Todo aprendizaje concebido dentro de este modelo supone, por tanto, una construcción que se 
realiza a través de un proceso mental que conlleva la adquisición de un conocimiento nuevo. El proceso concebido por esta corriente destaca no sólo esa reciente obtención sino la posibilidad de construirlo y adquirir una nueva competencia que le permite generalizar, es decir, aplicar lo previamente conocido a una situación nueva. El proceso anterior rechaza, por tanto, la idea de una asociación mecánica de estímulo-señal y supone una transformación significativa en el sujeto que lo lleva a cabo. Esta apreciación está estrechamente relacionada con los siguientes cuestionamientos: ¿quién conoce? ¿qué conoce? y ¿cómo conoce? (MÁYNEZ, 2009, p. 152).

Fray Bernardino de Sahagún (1499-1590) quien había adquirido su formación humanística en una de las instituciones académicas más acreditadas de Europa, la Universidad de Salamanca, estuvo durante más de sesenta años (15291590) en un continuo diálogo con los nativos del altiplano central de México: conversando con ellos en su propia lengua, la mexicana o náhuatl, y adoctrinándolos. Con sus alumnos del Colegio de Tlatelolco a los que impartió la cátedra de gramática y con los tlamatinime o sabios, emprendió la elaboración de un conjunto de obras sobre su historia y costumbres que quedó registrado en los distintos manuscritos que conforman su magna Historia General de las cosas de Nueva España (1546-1577) También con ellos elaboró, como él mismo lo admite, un conjunto de textos doctrinales indispensables para la tarea religiosa que tenía que desempeñar.

La develación lingüística del universo indígena que fray Bernardino de Sahagún consignó en la narración castellana del Códice Florentino (1577) - la única versión bilingüe y más completa de su Historia General ${ }^{5}$ fue, así, a través de la

\footnotetext{
${ }^{5}$ El Códice Florentino recibe este nombre debido que se ha conservado en la biblioteca MediceaLaurenziana de Florencia. Su elaboración se llevó a cabo entre 1575 y 1577 en el Colegio de Santa Cruz de Tlatelolco. Sahagún contó con el auxilio de sus escribanos indígenas.

La obra abarcó los doce libros de la Historia General. En la columna izquierda se incluyó el texto en náhuatl cuyo material fue recopilado primero desde 1553, antes de recibir la orden oficial del provincial Francisco Toral. Dicho apartado está dividido en libros y capítulos y, algunas veces, también en párrafos. En la columna derecha aparece la versión al castellano que no es literal sino una paráfrasis que resume y comenta, en ocasiones, el texto que le fue proporcionado por sus informantes indígenas. La obra se conformó inicialmente en cuatro tomos e incluyó un sinnúmero de aspectos propios de la vida, cultura y pensamiento de los nahuas del altiplano central: dioses, fiestas, cosmogonía, calendario discursos y adivinanzas, naturaleza, estratificación social y
} 
categorización occidental de la que procedía; de ahí que sea frecuente en la columna izquierda del Códice (lámina 1), la relación de correspondencia entre conceptos y objetos del viejo y nuevo mundos, mediante términos o frases del tipo, Ilaman o se llama, que es como, que parece, porque tiene semejanza, que quiere decir. El modelo, en estos casos, es A es $\mathbf{B}$ o $\mathbf{A}$ es como, o parece $\mathbf{B}$, en donde el término náhuatl puede aparecer antes o después de la palabra o paráfrasis castellana introducidas por las frases aclarativas de traducción. Algunos ejemplos los tenemos en: "A este propósito iban luego a buscar y a hablar al adivino que se llama tonalpouhqui, que quiere decir, "sabe conocer la fortuna de los que nacen" (SAHAGÚN, 2002, T.II, p.642).

Otros mandamientos o consejos daban la partera a la preñada para que los guardase entretanto duraba la preñez. Mandábala que no comiese aquel betún negro que se llama tzictli porque la criatura por esta causa no incurriese en el peligro que se llama netentzoponilixtli, y que no se hiciese el paladar duro y las encías gruesas, porque no podría mamar y si muriría (Las negritas son nuestras Ibid, p. 605).

Otras veces, fray Bernardino prefirió emplear términos que consideró absolutamente intercambiables, mediante la incorporación de la conjunción disyuntiva $\boldsymbol{o}$, la cual funciona también como aclaración del concepto al que alude ${ }^{6} \mathrm{o}$ identificación de lo que consideró dos mismas realidades amerindias:

[Cuando bautizaban a la criatura] "[...] Hacían también comida de mulli o potaje, con frisoles y maíz tostado" (Sahagún, op cit, T.II, p. 644).

[En la ceremonia del casamiento] "Muchas de las mujeres llevaban mantas y las ofrecían. Otras, que eran más pobres, ofrecían maíz. Todo esto ofrecían delante del fuego, y los viejos y viejas bebían uctli o pulcre, y bebían en unos vasos pequeños, templadamente" (La negrita es nuestra Ibid, p.584).

Recordemos que pulque o pulcre es un antillanismo, como lo son también los términos, cacique, canoa. En numerosas ocasiones, fray Bernardino proporcionó dos o más designaciones para aludir a lo que juzgó un mismo referente. El caso más claro es el de "Tezcatlipoca" que en el Libro Primero, y a lo largo del resto, es denominado mediante diferentes formas: Monenequi,

conquista, son algunos de ellos. Para mayor referencia al respecto, (vid., LEÓN-PORTILLA, 1999, p. 169-170).

${ }^{6}$ (Vid., SECO, 1972, p. 129).

caleidoscópio: linguagem e tradução / v. 2 / n. 1 [jan. - jun. 2018] p. 50-62 | ISSN: 2526-933X

Línguas Indígenas 
Moyocoyatzin, Necoc Yaotl, Nezahualpilli, Teimatini, Teyocoyani, Titlacahuan, que es la forma más frecuente, Yaotl, Yohualli Ehecatl. En ocasiones, incluso, explica la causa que motiva el nombre o atributo de esta deidad:

Y tenían que cuando andaba en la Tierra movía guerras, enemistades y discordias, de donde resultaban muchas fatigas y desasosiegos. Decían que el mesmo incitaba a unos contra otros para que tuviesen guerras y por eso le llamavan Nécoc Yáutl; qujere decir "sembrador de discordia, de ambas partes" (Ibid., T.I, p.71).

En cuanto [...] Yoalli ehecatl, "qujere decir tinebla y ayre, que está en todos lugares" (Ibid., T.II, p. 118r.).

Pero Sahagún, además, dejó un pormenorizado elenco designativo en determinados campos semánticos, como el relativo a la comida de los señores y a las mantas e insignias que usaban (Libro Octavo). Incluso, llegó a precisar el empleo de una forma lingüística en un sociolecto concreto. En la parte relativa a la creación del Sol y la Luna que corresponde al libro séptimo se refiere a Xólotl quien se rehusaba a morir, por eso se escondió "entre los maizales y volvióse y convertióse en pie de maíz que tiene dos cañas, y los labradores le llaman Xólotl" (Ibid., T.II, p. 697). Resulta significativo, asimismo, la profusa referencia y descripción las comidas y los atavíos propios de los señores que se incluyen en el Libro Octavo, en los que, en ocasiones, incluso, Sahagún incorpora su parecer

[...] comían también unas langostas que se llaman chapolín chichiahua; quiere decir "cazuela de unas langostas" y es muy sabrosa comida. Comían también unos gusanos que se llaman meocuilti chiltecpin mollo; quiere decir "gusanos que son de maguey y con chiltecpinmolli (Ibid., T.II, p.753). ${ }^{7}$

\section{Consideraciones finales}

Si bien es cierto que el lenguaje acota formalmente nuestro mundo, también es verdad, como resulta evidente en la pormenorizada relación designativa consignada por fray Bernardino de Sahagún en la versión parafrástica en español del Códice Florentino, que "El mundo se enriquece con cada nueva palabra; pues cada nueva palabra es una luz que alumbra algún rincón del mundo y torna visibles

7 Para mayor referencia acerca de los procedimientos empleados por el fraile para acercar y traducir a los europeos los nuevos conceptos y realidades del mundo indígena, consúltese (vid., Máynez, 2002, p. XLI-LII).

caleidoscópio: linguagem e tradução / v. 2 / n. 1 [jan. - jun. 2018] p. 50-62 | ISSN: 2526-933X

Línguas Indígenas 
cosas, pensamientos y sentimientos que antes yacían inadvertidos en la oscuridad", como afirma Stephen B. Presser (BOLLNOW, 1969, p. 106-107).

El primer contacto entre hombres de diferentes latitudes y culturas proveyó de ilimitados términos para el acercamiento a objetos y conceptos inimaginables y de su con siguiente apropiación a través de las explicaciones que fueron dando a partir de su particular acercamiento. La obra del franciscano Bernardino de Sahagún, sin duda la fuente más completa y consultada del México indígena, contiene el testimonio de la antigua palabra en lengua mexicana y de su transvase a un sistema conceptual y formal ajeno que permitió aprehenderla.

\section{REFERENCIAS}

\section{Fuentes:}

Durán, Diego. 1967. Historia de las Indias de Nueva España e Islas de la Tierra Firme, T. I, Ed. Ángel María Garibay, México: Porrúa [1575-1579].

Sahagún, Bernardino de, 1979. Códice Florentino. Historia general de las cosas de la Nueva España. Manuscrito 218-20 de la Colección Palatina de la Biblioteca Medicea Laurenziana, Edición facsimilar, 3 vols. México, Florencia: Casa Editorial Giunti Barbera. Archivo General de la Nación [1577].

2002. Historia General de las Cosas de Nueva España, T. I, Versión íntegra del texto castellano del manuscrito conocido como Códice florentino. Estudio introductorio, paleografía, glosario y notas de Alfredo López Austin y Josefina García Quintana, México: CONACULTA [1577].

\section{Estudios:}

Beattie, John. 1980. Otras culturas. Objetivos, métodos y realizaciones de la Antropología Social. México: Fondo de Cultura Económica.

Benveniste, Emile. 1982. "Estructuras de la lengua y la sociedad", en Problemas de lingüística general II, México: Siglo Veintiuno Editores.

Bollnow, Otto Friederich.1969. Hombre y espacio. Trad. Jaime López de Asiain y Martín, Madrid: Labor.

Foucault, Michel.1998. Las palabras y las cosas, una arqueología de las ciencias humanas. Trad. Elsa Cecilia Frost, 26a .ed. en español. México: Siglo Veintiuno Editores. 
Frost, Elsa Cecilia. 2002. La historia de Dios. Historia en las Indias. Visión franciscana del Nuevo Mundo. México: Tiempo de Memoria Tusquets Editores.

Glasersfeld, Ernest von 19 Dic.2017. "Constructivismo radical. Glaserfeld". Recuperado en https://jeasacademia.wordpress.com

León-Portilla, Miguel.1999. Bernardino de Sahagún. Pionero de la antropología. México: Universidad Nacional Autónoma de México y El Colegio Nacional.

Máynez, Pilar. 2002. El calepino de Sahagún. Un acercamiento. México. Universidad Nacional Autónoma de México y Fondo de Cultura Económica.

. 2009. "El proyecto lexicográfico de Bernardino de Sahagún en el proceso de comprensión de los dioses mexicas”, en Otto Zwartjes, Ramón Arzápalo y Thomas C. Smith Stark (eds.) Misionary Linguistics IV, Lingüística Misionera Lexicography.Amsterdam/Philadelphia. John Benjamins Publishing Company, 151165.

Ortega y Gasset, José. 1972. El hombre y la gente I, Madrid: Revista de Occidente (Colección el Arquero).

Pastrana Flores, Miguel. 2004. Historias de la Conquista. Aspectos de la Historiografía de Tradición Náhuatl. México: Universidad Nacional Autónoma de México.

Rosenblat, Ángel. 1977. “La hispanización de América. El castellano y las lenguas indígenas desde 1492". Los conquistadores y su lengua. Venezuela: Ediciones Caracas, pp. 91-136.

Seco, Manuel.1972. Gramática esencial del español, Introducción al estudio de la lengua. Madrid. Editorial Aguilar. 


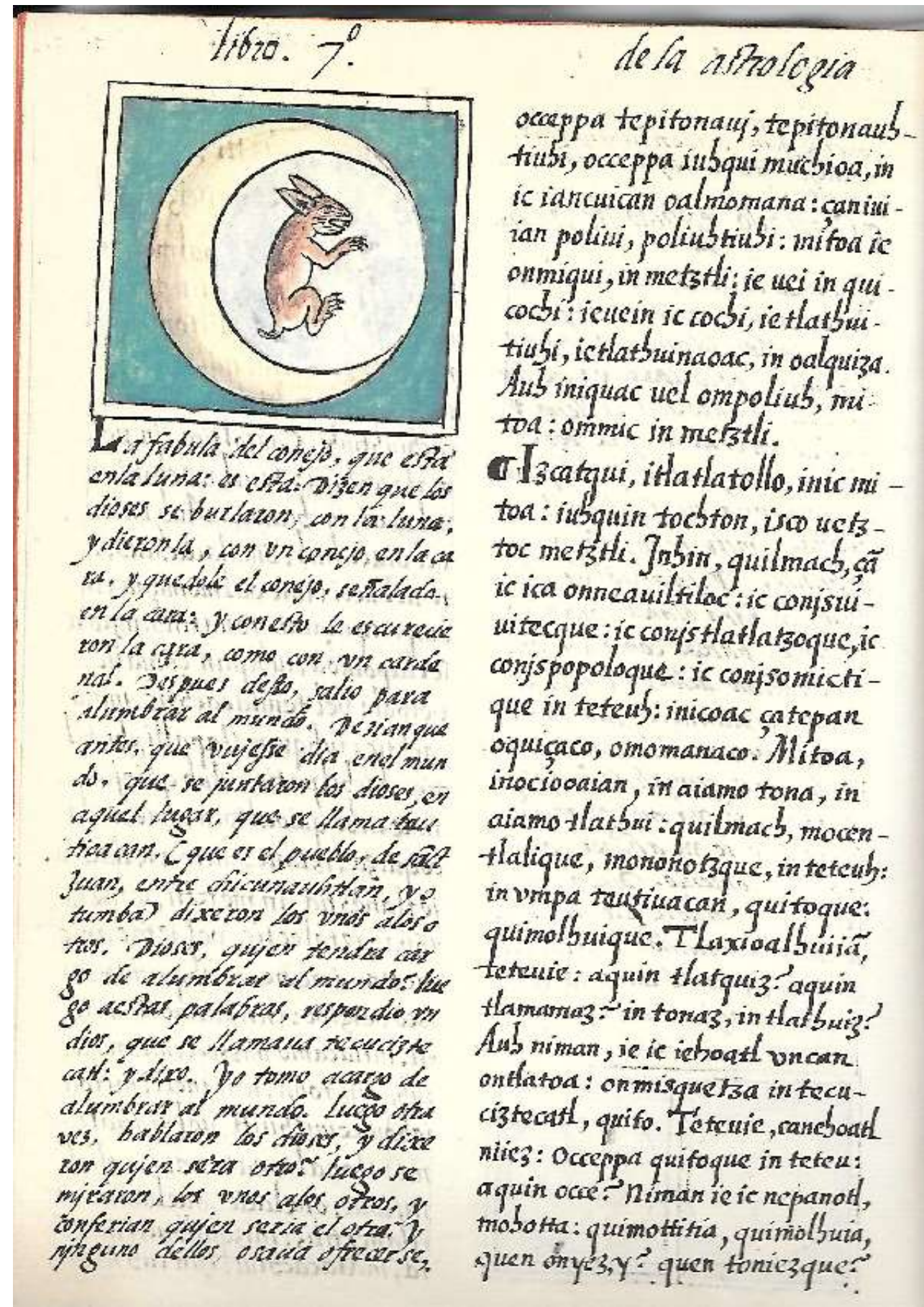

Códice Florentino. Historia general de las cosas de la Nueva España. Manuscrito 218-20 de la Colección Palatina de la Biblioteca Medicea Laurenziana, Edición facsimilar. T.II, Lib. 7, f. 2v., p. 228v.

\section{Biografía de la autora}


Pilar Máynez es Profesora de Carrera Titular "C" tiempo completo definitivo en la Facultad de Estudios Superiores Acatlán de la UNAM donde imparte las asignaturas de Teorías Lingüísticas I y II para la carrera de Lengua y Literatura Hispánicas y Lingüística General II para la Licenciatura en Enseñanza de Inglés desde 1981. Sistema Nacional: Nivel 2. PRIDE: Nivel “D” por cuatro periodos consecutivos.

Recebido em: 29/01/2018

Aprovado em: 08/03/2018

Publicado em junho de 2018 